\title{
Prostate cancer chemopreventive activity of phenethyl isothiocyanate through epigenetic regulation (Review)
}

\author{
LONG G. WANG ${ }^{1}$ and J.W. CHIAO ${ }^{2}$ \\ ${ }^{1}$ NYU Cancer Institute, New York University School of Medicine, New York, NY 10010; \\ ${ }^{2}$ Department of Medicine, New York Medical College, Valhalla, NY 10595, USA \\ Received February 1, 2010; Accepted April 6, 2010
}

DOI: 10.3892/ijo_00000702

\begin{abstract}
Prostate cancer is one of the most commonly diagnosed cancers in men. The number of affected men is expected to rapidly increase as the population of males over the age of 50 grows worldwide. For patients who are not cured by local treatment and experience metastatic disease, neither androgen ablation nor chemotherapy can abrogate progression and death from androgen-independent/hormonerefractory disease. Therefore, finding strategies for the prevention of prostate cancer initiation and disease progression is a medical challenge. Consumption of cruciferous vegetables has been reported to be associated with reduced incidence of prostate cancer cases. The isothiocyanates, including phenethyl isothiocyanate (PEITC), from cruciferous vegetables have been demonstrated as active components responsible for chemoprevention. In this review, we summarize the recent findings of PEITC on prostate cancer prevention with an emphasis on epigenetic mechanisms. Studies have indicated that PEITC mediates gene regulation, such as downregulation of androgen receptor expression and induction of endogenous cyclin-dependent kinase inhibitors, p21 and p27. The gene for detoxifying enzyme $\pi$-class glutathione S-transferase (GSTP1), silenced in the vast majority of prostate tumor cells, could be reactivated and the enzymatic function recovered. This may be through epigenetic mechanisms as PEITC is a dual inhibitor of histone deacetylases and aberrant $\mathrm{CpG}$ island methylation of various genes. The epigenetic regulation may play a critical role, along with interactive mechanisms including the disruption of microtubule polymerization, in prostate cancer prevention by PEITC. These mechanisms
\end{abstract}

Correspondence to: Dr Long G. Wang, NYU Cancer Institute, New York University School of Medicine, Manhattan VAMC, 18th Floor, Room 18003W, 423 East 23rd Street, New York, NY 10010, USA

Dr J.W. Chiao, Department of Medicine, New York Medical College, Vosburgh 207, Valhalla, NY 10595, USA

E-mail: longgui.wang@nyumc.org; jen-wei_chiao@nymc.edu

Key words: prostate cancer, phenethyl isothiocyanate, epigenetics, histone acetylation target and correct the aberrations fundamental to the initiation and progression of carcinogenesis in cells, and restoring the cells to a more normal state. Inhibiting and eliminating cancer cells forms the basis of cancer prevention.

\section{Contents}

1. Introduction

2. Consumption of Brassica vegetables reduces prostate cancer risk

3. Downregulation of AR expression by PEITC through epigenetic mechanisms

4. Restoration of GSTP1 expression by PEITC via $\mathrm{CpG}$ island demethylation

5. PEITC inhibits prostate tumors and CGI methylation of the MGMT gene in TRAMP mice

6. Induction of endogenous cdk inhibitors by PEITC

7. Summary and conclusions

\section{Introduction}

Prostate cancer is the most commonly diagnosed cancer in men in the US. There were an expected 192,280 new cases and 27,360 deaths from prostate cancer in 2009 (1). Prostate cancer is usually found in elderly men. The prevalence of prostatic intraepithelial neoplasia and proliferative inflammatory atrophy, which are believed to be precursors of prostate cancers, may have a long latency period of ten or more years before developing into invasive carcinomas $(2,3)$. Such factors lend credence to the growing belief that any delay in the time course of neoplastic development, achieved through pharmacological, hormonal and nutritional intervention, could result in a substantial reduction in the incidence of tumors. Even a modest reduction in the slope of the cancer latency curve could delay the onset and the effects of prostate cancer in individuals at risk for the disease. In addition, the limited success of current treatments for most advanced prostate cancer highlights the importance of cancer prevention. Therefore, the development of strategies for the prevention of prostate cancer appears to be a practical goal that may lead to a reduction in the incidence, morbidity and mortality associated with the disease. As such, more than a dozen classes of natural and synthetic novel agents are in clinical evaluation and hold 
promise for preventing the development of clinically aggressive prostate cancers $(3,4)$.

In addition to genetic alteration, epigenetic mechanisms have recently been described as important factors contributing to the carcinogenesis and progression of prostate cancer. Epigenetic regulation, defined as heritable changes in gene expression that occur without changes in DNA sequence, include DNA hypermethylation and hypomethylation, and histone modifications. In this review we attempt to summarize the findings concerning isothiocyanates, which are present naturally in cruciferous vegetables, in particular phenethyl isothiocyanate (PEITC), in prostate cancer prevention and their role in epigenetic regulation.

\section{Consumption of Brassica vegetables reduces prostate cancer risk}

There is epidemiological evidence that the incidence of prostate cancer varies in different regions of the world. As populations move to high-risk areas, they assume the risk of that geographic region (5-8). Substantial differences exist in age-adjusted incidence and mortality between East Asia, Northwest Europe and North America. However, these differences are not caused predominantly by genetic differences between populations; therefore, one may infer a relationship of environment and diet to the cancer risk.

A review by Kristal and Lampe (9) indicated that the epidemiological literature provides modest to strong support for the hypothesis that a high intake of Brassica vegetables reduces prostate cancer risk. The crucifereous vegetables include the Brassica family, and examples are broccoli, cabbage, Brussels sprouts, kale, mustard, watercress and cauliflower. One of the epidemiological studies involved a populationbased case-control study of men under 65 years of age. This study showed that three or more servings of cruciferous vegetables per week was a significant factor in reducing prostate cancer cases as compared to that of one serving or less per week (10). Another study involved a multicenter casecontrol study of African-American, Caucasian, Japanese and Chinese men. The results of this study convincingly showed that an intake of cruciferous vegetables was inversely related to prostate cancer cases, particularly to advanced cases ( $\mathrm{P}$ for trend $=0.006$ ). The findings were generally consistent across ethnic groups (11). The conclusions suggest that the effects may not only be limited to preventing the initiation of prostate cancer, but may also inhibit the progression of the disease.

Isothiocyanates have been identified as the active components present in Brassica vegetables that act against prostate cancer. The isothiocyanates occur naturally as thioglucoside conjugates, i.e., glucosinolates, in a wide variety of cruciferous vegetables (12). Isothiocyanates are released from glucosinolates by the hydrolytic action of the enzyme myrosinase when the plant tissues are crushed or masticated (13). Hydrolysis of the glucosinolate, gluconasturtin, found in high amounts in watercress, yields phenethyl isothiocyanate (PEITC). Isothiocyanates have been shown to have potent cancer chemopreventive activity in several experimental models as well as in humans. These models include cancers of the lung, mammary gland, esophagus, liver, pancreas, fore-stomach, small intestine, colon and bladder of mice, rats, and other rodents and colon cancer in humans (14-17). Initially, the modes of action of isothiocyanates have been described as inhibitors of phase I enzyme CYP450 and inducers of phase II detoxification enzymes such as glutathione S-transferase and $\mathrm{NAD}(\mathrm{P}) \mathrm{H}$ guinone reductase, thereby blocking the metabolic activation of procarcinogens and facilitating carcinogen excretion. Chiao et al provided the first report demonstrating that isothiocyanates and their metabolites can mediate other functions including growth inhibition and apoptosis induction in prostate cancer cells, indicating that they are also effective at the post-initiation progression stages of carcinogenesis (18).

\section{Downregulation of AR expression by PEITC through epigenetic mechanisms}

By far the most extensively studied transcriptional factor in prostate cancer is the androgen receptor (AR). Abnormalities of AR expression and activity in prostate cancer are caused by both genetic and epigenetic mechanisms. Genetic alterations include point mutations at transcriptional activation or the ligand-binding domains and gene amplifications, which are not the major focus of this review. Epigenetic mechanisms comprise changes in AR transcription due to $\mathrm{CpG}$ island hypermethylation and changes in AR protein levels caused by altered posttranslational degradation. In addition, epigenetic changes also occur in AR signaling through its coactivators or corepressors, or its interacting partners of other transcriptional factors that are altered epigenetically.

In pathological analyses, a significant loss of AR expression has been observed in prostate tumors for which AR methylation may be accountable. It has been reported that methylation of several consensus sequences in the AR minimal promoter region are strongly associated with the loss of AR expression in metastatic hormone-independent prostate cancer cell lines $(19,20)$. In clinical tumor tissues, the silencing of AR was also found to correlate with hypermethylation of the AR promoter. While there was no methylation in normal and primary prostate cancers that expressed the AR, $27 \%$ of tumors obtained from men who died from hormone refractory disease demonstrated a significant loss of AR expression, and $50 \%$ of these AR-negative tumors had a methylated AR (19).

In contrast, the common denominator in prostate cancer progression for the vast majority of cases is sustained or increased expression and activity of the AR in androgendeprived conditions (21-23). While genetic mechanisms such as AR gene mutation and amplification of the wild-type AR have been demonstrated to be involved, epigenetic alterations also have a critical role in elevated AR activities.

Gao et al recently established an androgen-independent (AI) sub-line of prostate carcinoma $\mathrm{LNCaP}$ that strongly overexpresses the AR (24). These ARs are functional, phosphorylated and, at a high level, inhibit the expression of the $\mathrm{p} 21^{\mathrm{WAF} / \mathrm{CIPI}}$ gene (25). Such activity may promote hormoneindependent growth and resistance to apoptosis in response to chemotherapeutic agents $(24,25)$. Using this cell line, a novel AR suppressor sequence (ARS) in the 5'-untranslated region of the AR gene was identified, and the accumulated data, to-date, demonstrated the existence of a repressor(s) (suppressor) that controls normal AR expression. Loss of this 
suppressor expression may be the basis of the switch of prostate cancer cells from a hormone dependent (AD) to an independent phase (AI). Significantly, it has been found that this suppressor protein(s) could be induced to re-express in AI cells through histone deacetylase inhibitors, such as trichostatin A, sodium butyrate or suberoylanilide hydroxamic acid (SAHA). Consequently, a normal AR function was restored as indicated by regaining an androgen-dependent phenotype and apoptotic sensitivity to chemotherapeutic agents (24-27). Consistent with these findings, histone deacetylase inhibitors LAQ824 and SAHA have also been reported to repress $A R$ at both the transcriptional and posttranslational levels $(28,29)$.

Moreover, it has been found that the AR is controlled epigenetically at the posttranslational level. AR belongs to a superfamily of nuclear receptors that requires the action of molecular chaperones for folding and hormone binding (30). The C-terminal Hsp-interacting protein (Chip) is a cochaperone that interacts with Hsp70 and Hsp90 molecular chaperones via a tetratricopeptide domain and inhibits chaperone-dependent protein folding in vitro. Chip was also found to stimulate protein degradation by acting as an E3 ubiquitin ligase via a modified ring finger domain called a $\mathrm{U}$ box. Chip overexpression was found to lead to a large decrease in AR steady state levels and increased levels of AR ubiquitinylation (31). Chen et al (29) reported that, while the histone deacetylase inhibitor LAQ824 repressed AR transcription, LAQ824 also stimulated AR degradation via inactivation of the Hsp90 molecular chaperone. The inactivation by LAQ824 was due to an enhanced acetylation of Hsp90, thereby inhibiting its ATP binding activity.

PEITC as HDAC inhibitor. We showed that, similar to other isothiocyanates such as sulforaphane (32), PEITC also is a histone deacetylase inhibitor $(33,34)$. Exposure of prostate cancer LNCaP cells to PEITC reduced the activity and levels of histone deacetylases (HDACs) and induced selective histone acetylation and methylation changes for chromatin unfolding. Compared to untreated control cells, a greater than 2 -fold increase in acetylated histone $\mathrm{H} 3$ was noted with $1 \mu \mathrm{M}$ PEITC. PEITC selectively increased the level of mono/di/ trimethylation at lysine 4 of histone $\mathrm{H} 3$ while decreasing the level of trimethylated lysine 9 of H3. The effect of PEITC for decreasing methylation at $\mathrm{H} 3 \mathrm{~K} 9$ was found to be stronger than that of sodium butyrate, a known HDAC inhibitor used as a control.

Consequently, similar to other histone deacetylase inhibitors $(28,29)$, PEITC significantly downregulated the $\mathrm{AR}$ at both the transcriptional and posttranslational levels (35). It repressed AR transcription via inhibition of the transcription factor $\mathrm{Sp} 1$ and reduced AR protein by accelerating protein degradation. As a result, PEITC induced a significant growth inhibition with equal $\mathrm{IC}_{50}$ in both LNCaP AD and LNCaP AI cells.

\section{Restoration of GSTP1 expression by PEITC via CpG island demethylation}

Transcriptional silencing of genes due to hypermethylation of $\mathrm{CpG}$ islands in the promoter region of genes has been reported in nearly every type of human tumor. Hypermethylation and silencing of genes involved in DNA repair (e.g. MGMT and hMLH1) or detoxification (GSTP1) result in DNA mutation or inflammation (36-38). A broad spectrum of other genes are frequently hypermethylated in cancers, including those associated with cell cycle regulation, apoptosis, angiogenesis, and invasion and metastasis $(36,37,39-42)$.

Prostate cancer is characterized by the loss of expression of the $\pi$-class glutathione-S-transferase (GSTP1), which encodes a detoxifying phase II enzyme facilitating the removal of toxins. More than $95 \%$ of clinical prostate tumors lack GSTP1, which is considered as a risk factor for the disease $(43,44)$. Methylation of other genes, including RARß2, RARß4, RASSF1A, CDH13, APC, CDH1 and FHIT has also been reported in prostate cancers $(45,46)$. We recently demonstrated that several genes, including p21, Bax, PSA and the AR suppressor (ARS) were repressed in androgenindependent but not in androgen-dependent cells. The silencing of PSA and ARS has been shown to be the likely result of CpG island methylation $(25,27)$. The silencing of p21, Bax and ARS may also be associated with both the drug resistance of cancer cells to chemotherapeutic agents and the androgen-independent progression of the disease $(25,27,47)$. Thus, restoration of the expression of silenced genes, via demethylation of the $\mathrm{CpG}$ island, may correct the aberrations associated with carcinogenesis and may prevent androgenindependent progression.

PEITC has been recognized as an inducer of phase II detoxification enzymes, GSTs $(48,49)$. To investigate the mechanism of PEITC to induce GSTP1, LNCaP AD and its sub-line LNCaP AI $(24,25,27)$ were exposed to PEITC. The status of the methylated and the unmethylated forms of GSTP1 was measured by methylation-specific PCR (MSP) $(50,51)$ and quantified by pyrosequencing. The cell lines were also exposed to a DNA methylation inhibitor 5-Aza-2'-deoxycytidine (5'-Aza) which is capable of restoring the expression of GSTP1 by demethylation $(38,46)$. As expected, no basal level of the unmethylated form of $\mathrm{CpG}$ island methylation of the GSTP1 was detected in both AD and AI cells by MSP. However, it became detectable after exposure of the cells to PEITC (1.0-2.0 $\mu \mathrm{M})$ in a concentration-dependent manner. The demethylation effects of PEITC were similar to those after exposure to 5'-Aza. Pyrosequencing analysis showed that, in comparison with normal prostate tissues, the $\mathrm{CpG}$ island at two indicated positions was heavily methylated in $\mathrm{AD}$ cells (C: 89.5 and $61.8 \%$ respectively) (33). After exposure to $2 \mu \mathrm{M}$ of PEITC for 5 days, however, the methylated $\mathrm{CpG}$ island at both positions was significantly decreased from 89.5 to $73.2 \%$ at position 1 , and from 61.8 to $6.5 \%$ at position 2 . The effect of PEITC-mediated demethylation was found to be stronger than that of 5'-Aza $(5 \mu \mathrm{M})$ under the same experimental conditions. After exposure to $5 \mu \mathrm{M}$ of 5 '-Aza for 5 days, the methylated CGI deceased from 89.5 to $78.3 \%$ at position 1, and from 61.8 to $37.7 \%$ at position 2 in AD cells. Consequently, a significant increase in the level of GSTP1 protein and $>5$-fold enzyme activity was observed in both $\mathrm{AD}$ and AI cells after exposure to $1 \mu \mathrm{M}$ of PEITC for 5 days. Thus, our data indicate that, similar to 5'-Aza, PEITC is a potent methylation inhibitor, not only in androgen-dependent, 
A

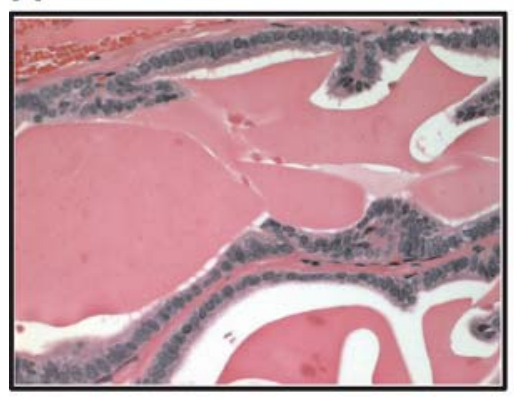

D

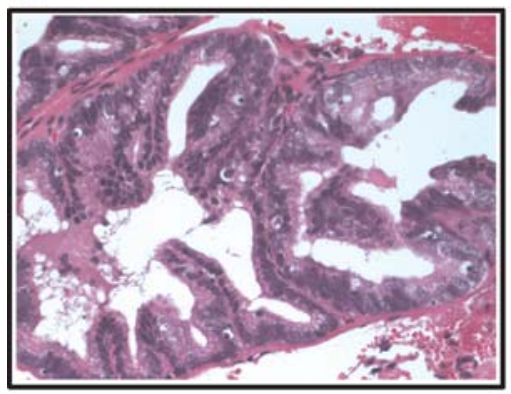

B

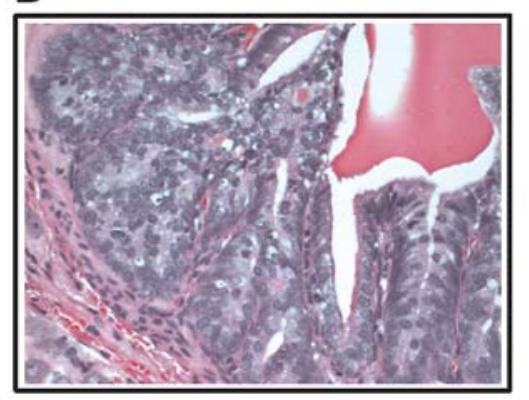

E

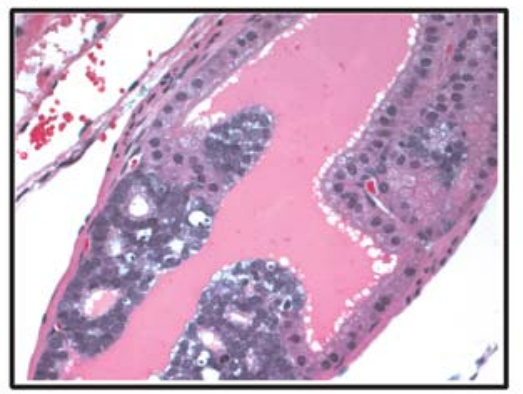

C

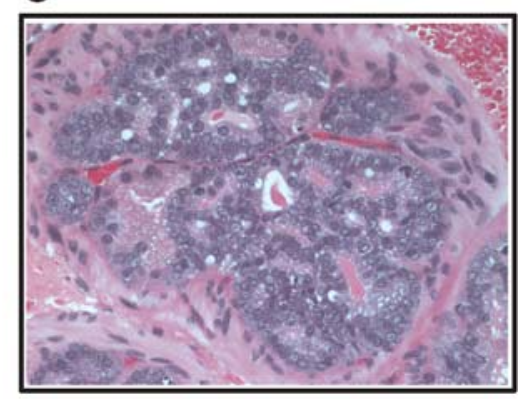

F

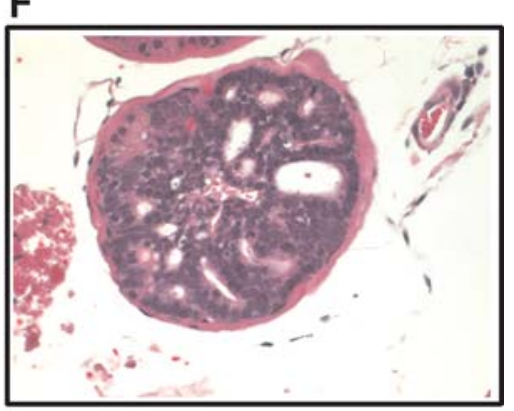

G

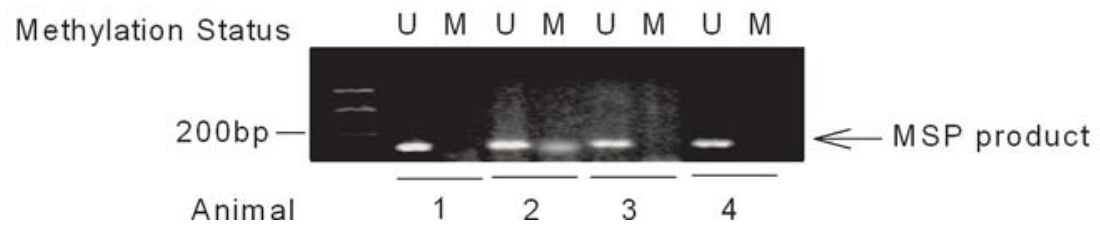

Figure 1. Morphology of prostate sections of TRAMP mice stained with H\&E. Representative images of prostate sections from untreated mice are presented in A-C. (A) A normal mouse prostate section with a prostatic gland lined with a single layer of cuboidal epithelial cells. (B) The presence of high-grade PIN with papillary projections lined with epithelial cells at the lower right corner, and foci of adenocarcinoma arising from a background of PIN (upper left corner). (C) Well-differentiated adenocarcinoma characterized by cribriform architecture. Representative images of prostate sections from PEITC-treated mice are presented in D-F. (D) Focal adenocarcinoma arising from a background of PIN. (E) Carcinoma gland located adjacent to normal epithelium. (F) Gland with cribriform structure with well-differentiated adenocarcinoma. Magnification, x400. (G) CGI methylation of the MGMT gene promoter by MSP. DNA was extracted from the prostates of wild-type C57 mice (1), untreated (2) or two PEITC-treated TRAMP mice (3 and 4), using the Trizol DNA isolation kit. DNA $(0.5 \mu \mathrm{g})$ was used for the MSP according to the procedures described by Yamada et al (51). The primers for the methylated form: 5'-GGT AGT TTT TAG AGT TAC GTT TCG CGT-3' and 5'-CAA ACG CGT ACA CGA AAT AAA AAC GAA A-3'; and for the unmethylated form: 5'-TTT GGT AGT TTT TAG AGT TAT GTT TTG TGT-3' and 5'-CCA CAA ACA CAT ACA CAA AAT AAA AAC AAA-3' were used. M: methylated form; U: unmethylated form.

but also in androgen-independent cells, which reactivates and restores the function of the GSTP1 gene (33).

\section{PEITC inhibits prostate tumors and CGI methylation of the MGMT gene in TRAMP mice}

We evaluated whether PEITC is a potential agent for preventing and inhibiting prostate cancer using a model of TRAMP transgenic mice which form spontaneous prostate tumors. The experimental group of mice, each 6 weeks old, was administered $15 \mu \mathrm{mol}$ PEITC prepared in corn oil daily by gavage for 13 weeks. The control group received corn oil without PEITC as a vehicle control.

During the experimental period and at necropsy, the body weight of the experimental mice did not show a greater than $10 \%$ difference from the control group. The weight of spleens and livers from PEITC-treated and untreated mice were nearly identical, suggesting that there was no overt toxicity with the PEITC feeding.
At necropsy after 13 weeks of feeding, multiple sections of the prostate lobes of the TRAMP mice were prepared and examined by an independent pathologist. Among the untreated mice, $70 \%$ of the prostate glands were normal. They were typically lined by a single layer of cubiodal epithelial cells with small nuclei and inconspicuous nucleoli (Fig. 1A). Ten percent of the glands demonstrated high-grade prostatic intraepithelial neoplasia (PIN) with papillary projections lined by epithelial cells with prominent nucleoli (Fig. 1B, lower right corner). In addition, foci of adenocarcinoma arising from a background of PIN were evident (upper left corner). Twenty percent of the examined glands revealed well-differentiated adenocarcinoma characterized by cribriform architecture, packed with small crowded glands with neoplastic cells characterized by somewhat vesicular nuclei and small prominent nucleoli (Fig. 1C). Among the prostate glands from the PEITC-treated mice, 93\% were normal. Five percent of the glands showed focal adenocarcinoma, with $3.8 \%$ arising from a background of PIN (Fig. 1D). In contrast to 
the mice without PEITC treatment, some carcinoma glands were located adjacent to normal epithelium (Fig. 1E). Only $2 \%$ of the glands demonstrated cribriform structure with adenocarcinomas (Fig. 1F). These results demonstrated that PEITC is effective in inhibiting the development of prostate tumors in TRAMP mice.

The status of CGI methylation of the DNA repair gene $\mathrm{O}(6)$-methylguanine-DNA methyltransferase (MGMT) was examined, using DNA extracted from prostate tissues of TRAMP mice treated or untreated with PEITC, or from wild-type C57BL mice used as normal controls. Upon MPS, (Fig. 1G) methylated MGMT was only noted in the untreated mice, and not in the normal controls or PEITC-treated mice. This indicated that the CGI hypermethylation occurring at the early stage of prostate carcinogenesis could be completely inhibited with PEITC treatment.

Overexpression of DNA methyltransferase 1 (DNMT1), which enzymatically methylates DNA in mouse embryonic stem cells, is capable of inducing genomic DNA hypermethylation and the loss of imprinting (52). To analyze the basis of inhibiting DNA hypermethylation by PEITC, the effects of dietary feeding of PEITC on the expression of DNMT1 in a cyproterone and testosterone-primed Wistar rat model was evaluated. In the testosterone-primed prostates, cellular proliferation was stimulated with a significant increase in the cyclins D1 and E, cdk2 and a decrease in p27. Concomitantly, $\mathrm{Rb}$ was inactivated as shown by an increase in $\mathrm{Rb}$ phosphorylation (54). Inactivation of $\mathrm{Rb}$ leads to enhanced activity of E2F1, thereby activating downstream target DNMT1 transcription. This is because DNMT1 is complexed directly with $\mathrm{Rb}, \mathrm{E} 2 \mathrm{~F} 1$ and HDAC1 to mediate DNA methylation, and the promoter of DNMT1 is regulated by $\mathrm{Rb} / \mathrm{E} 2 \mathrm{~F} 1$ (53). Consequently, the expression of DNMT1 was clearly enhanced in the prostates after testosterone priming. Both the enhancement of DNMT1 and proliferation were negated by the feeding of PEITC at $5 \mathrm{mg} / \mathrm{kg}$, revealing a mechanism of inhibiting DNA methylation.

\section{Induction of endogenous cdk inhibitors by PEITC}

Tumorigenesis is characterized by aberrant regulation of cell proliferation due to a chronic loss of the integration and coordination of extracellular signals within the cell cycle machinery. While aberrantly elevated activity of cyclindependent kinases (cdks) is a common defect in human cancers, endogenous cdk inhibitors, such as p16, p21 and p27 are often epigenetically silenced, which have recently been implicated in the development of hormone refractory prostate cancer $(55,56)$. Induction of the cdk inhibitors is associated with the growth inhibition of hormone refractory prostate cancer and restored responses to hormone- and chemotherapies $(25,57)$.

The strong genetic and epigenetic link between cdks and carcinogenesis has provided the rationale for developing small-molecule inhibitors of cdks, for both treatment and prevention of cancers (58). Bakshi et al (59) explored the signaling mechanism of a unique natural antioxidant derived from spinach extract (NAO) in the chemoprevention of prostate cancer. Using human PC3 prostate cancer cells they found that NAO and two derived components achieved a

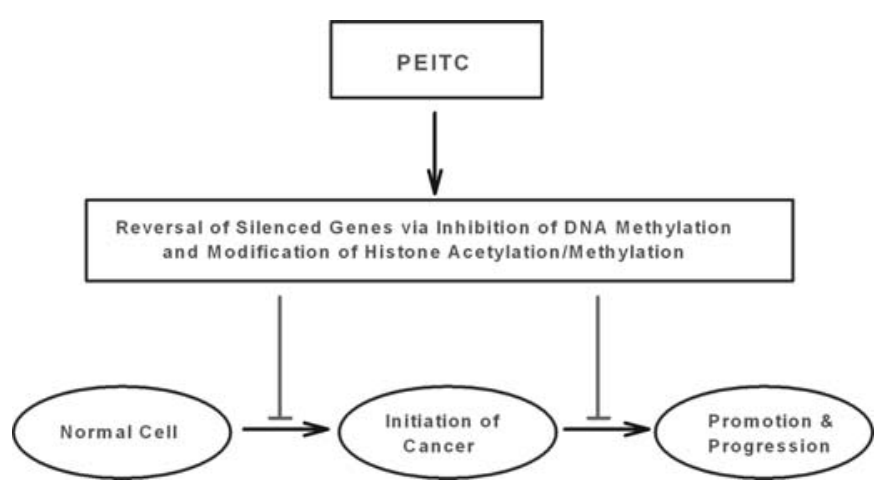

Figure 2. Proposed mechanism of phenethyl isothiocyanate (PEITC) in the prevention of prostate cancer.

significant increase in the p21 level while the expression of cyclin A and cdk-2 was reduced.

We described that isothiocyanates and their metabolites inhibit the levels and activities of cdk/cyclins and activate the cdk inhibitor p21 in prostate cancer cells $(60,61)$. To elucidate the mechanisms, we explored the epigenetic regulation on chromatin remodeling. Chromatin immunoprecipitation assay showed that acetylation of histone is associated with TATA box of the p21 promoter after PEITC treatment. In parallel, PEITC significantly enhanced histone acetylation and induced selective modification of histone methylation for chromatin remodeling. These observations strongly suggest that the upregulation of p21 transcription was likely due to an increased accessibility of the transcription factors to the p21 promoter. Additionally, PEITC was found to significantly inhibit the expression of c-Myc, which served as a repressor of p21 transcription. Pull-down assays using Sp1 affinity oligo beads of the $\mathrm{p} 21$ promoter showed that there was a decreased c-Myc binding to the $\mathrm{Sp} 1$ transcriptional complexes in the p21 promoter, resulting in reduced p21 repression (34).

\section{Summary and conclusions}

Inhibition of CpG DNA methylation in the promoter and modification of histone acetylation/methylation as cytoprotective mechanisms. Isothiocyanates, including PEITC, are potent chemopreventive agents. One well-documented mechanism involves the alteration of carcinogen metabolism and facilitation of their excretion, thus protecting cells from the assaults of carcinogens (62). Isothiocyanates have since been demonstrated capable of inhibiting post-initiation progression of carcinogenesis. They mediate growth arrest and apoptosis in cancer cells (18), and some isothiocyanates bind directly to tubulins, disrupting microtubule polymerization and inducing mitotic arrest (63). Multiple and interactive mechanisms, including epigenetic regulation, may be involved in the preventive and therapeutic effects of PEITC on cancers. Gene silencing through hypermethylation is mediated by a series of events, which include methylation of cytosines within the gene promoter and the establishment of heterochromatins in which the histone tails are modified through acetylation, phosphorylation, methylation and ubiquitylation $(64,65)$. The fact that pharmacological agents can reverse the process of 
epigenetically mediated gene silencing makes it an ideal target for prevention. Moreover, synergistic re-expression of silenced genes can be achieved by a combination of DNA demethylation and histone deacetylase inhibition (6). We recently demonstrated, for the first time to our knowledge, that PEITC reverses CGI hypermethylation of the GSTP1 gene and restores its detoxifying function in human prostate cancer cells while significantly inhibiting histone deacetylases and enhancing acetylation of histones (33). Unlike the known inhibitors for DNA methylation or HDACs that modify either DNA or histones, but not both, PEITC inhibits DNA methylation and HDAC concurrently. The simultaneous actions provide unique crosstalk between the DNA and chromatins. The reactivation of GSTP1, which is otherwise silenced in the vast majority of prostate tumors, has revealed a novel mechanism unique to prostate cancer chemoprevention and chemotherapy. This hypothesis is schematically presented in Fig. 2.

Unique also to prostate cancer is that PEITC downregulates the transcriptional factor $\mathrm{Sp} 1$, a regulator of $\mathrm{AR}$ expression. Lowered AR expression reduces testosterone activity, attenuating the growth of prostate cancer cells. Together, these findings reveal the effects of PEITC in targeting and correcting the aberrations that are fundamental to the initiation and progression of prostate carcinogenesis. They illustrate the basis of prostate cancer prevention by isothiocyanates and cruciferous vegetables.

\section{Acknowledgements}

This study was supported by a prostate cancer research grant from the US Army DAMD17-03-1-011.

\section{References}

1. Jemal A, Siegel R, Ward E, Hao Y, Xu J and Thun MJ: Cancer statistics, 2009. CA Cancer J Clin 50: 225-249, 2009.

2. Sakr WA, Haas GP, Cassin BF, Pontes JE and Crissman JD: The frequency of carcinoma and intraepithelial neoplasia of the prostate in young male patients. J Urol 150: 379-385, 1993

3. Lieberman R, Bermejo C, Akaza H, Greenwald P, Fair W and Thompson I: Progress in prostate cancer chemoprevention: modulators of promotion and progression. Urology 58: 835-842, 2001.

4. Cohen LA: Nutrition and prostate cancer: a review. Ann NY Acad Sci 963: 148-155, 2002.

5. Muir CS, Nectoux J and Staszewski J: The epidemiology of prostatic cancer. Geographical distribution and time-trends Acta Oncol 30: 133-140, 1991.

6. Belinsky SA, Klinge DM, Stidley CA, et al: Inhibition of DNA methylation and histone deacetylation prevents murine lung cancer. Cancer Res 63: 7089-7093, 2003.

7. Graham S, Haughey B, Marshall J, et al: Diet in the epidemiology of carcinoma of the prostate gland. J Natl Cancer Inst 70: 687-692, 1983.

8. Shimizu H, Ross RK, Bernstein L, Yatani R, Henderson BE and Mack TM: Cancers of the prostate and breast among Japanese and white immigrants in Los Angeles County. Br J Cancer 63: 963-966, 1991.

9. Kristal AR and Lampe JW: Brassica vegetables and prostate cancer risk: a review of the epidemiological evidence. Nutr Cancer 42: 1-9, 2002.

10. Cohen JH, Kristal AR and Stanford JL: Fruit and vegetable intakes and prostate cancer risk. J Natl Cancer Inst 92: 61-68, 2000.

11. Kolonel LN, Hankin JH, Whittemore AS, et al: Vegetables, fruits, legumes and prostate cancer: a multiethnic casecontrol study. Cancer Epidemiol Biomarkers Prev 9: 795-804, 2000 .
12. Fenwick GR, Heaney RK and Mullin WJ: Glucosinolates and their breakdown products in food and food plants. Crit Rev Food Sci Nutr 18: 123-201, 1983.

13. Sones HR and Fenwick GR: An estimate of the mean daily intake of glucosinolate from cruciferous vegetables in the UK. J Sci Food Agr 35: 712-715, 1984.

14. Chung FL, Morse MA, Eklind KI and Lewis J: Quantitation of human uptake of the anticarcinogen phenethyl isothiocyanate after a watercress meal. Cancer Epidemiol Biomarkers Prev 1: 383-388, 1992.

15. Hecht SS: Chemoprevention by isothiocyanates. J Cell Biochem Suppl 22: 195-209, 1995.

16. Khor TO, Cheung WK, Prawan A, Reddy BS and Kong AN: Chemoprevention of familial adenomatous polyposis in Apc (Min/+) mice by phenethyl isothiocyanate (PEITC). Mol Carcinog 47: 321-325, 2008.

17. Moy KA, Yuan JM, Chung FL, et al: Urinary total isothiocyanates and colorectal cancer: a prospective study of men in Shanghai, China. Cancer Epidemiol Biomarkers Prev 17: 1354-1359, 2008

18. Chiao JW, Chung F, Krzeminski J, et al: Modulation of growth of human prostate cancer cells by the $\mathrm{N}$-acetylcysteine conjugate of phenethyl isothiocyanate. Int J Oncol 16: 1215-1219, 2000.

19. Kinoshita H, Shi Y, Sandefur C, et al: Methylation of the androgen receptor minimal promoter silences transcription in human prostate cancer. Cancer Res 60: 3623-3630, 2000.

20. Nakayama T, Watanabe M, Suzuki H, et al: Epigenetic regulation of androgen receptor gene expression in human prostate cancers. Lab Invest 80: 1789-1796, 2000.

21. Taplin ME and Balk SP: Androgen receptor: a key molecule in the progression of prostate cancer to hormone independence. J Cell Biochem 91: 483-490, 2004.

22. Santos AF, Huang H and Tindall DJ: The androgen receptor: a potential target for therapy of prostate cancer. Steroids 69: 79-85, 2004.

23. Heinlein CA and Chang C: Androgen receptor in prostate cancer. Endocr Rev 25: 276-308, 2004

24. Gao M, Ossowski L and Ferrari AC: Activation of Rb and decline in androgen receptor protein precede retinoic acidinduced apoptosis in androgen-dependent $\mathrm{LNCaP}$ cells and their androgen-independent derivative. J Cell Physiol 179: 336-346, 1999.

25. Wang LG, Ossowski L and Ferrari AC: Overexpressed androgen receptor linked to $\mathrm{p} 21^{\mathrm{WAF} 1}$ silencing may be responsible for androgen independence and resistance to apoptosis of a prostate cancer cell line. Cancer Res 61: 7544-7551, 2001.

26. Wang LG, Johnson EM, Kinoshita Y, et al: Androgen receptor overexpression in prostate cancer linked to Pur alpha loss from a novel repressor complex. Cancer Res 68: 2678-2688, 2008.

27. Wang LG, Ossowski L and Ferrari AC: Androgen receptor level controlled by a suppressor complex lost in an androgenindependent prostate cancer cell line. Oncogene 23: 5175-5184, 2004.

28. Marrocco DL, Tilley WD, Bianco-Miotto T, et al: Suberoylanilide hydroxamic acid (vorinostat) represses androgen receptor expression and acts synergistically with an androgen receptor antagonist to inhibit prostate cancer cell proliferation. Mol Cancer Ther 6: 51-60, 2007.

29. Chen L, Meng S, Wang H, et al: Chemical ablation of androgen receptor in prostate cancer cells by the histone deacetylase inhibitor LAQ824. Mol Cancer Ther 4: 1311-1319, 2005.

30. Toft DO: Recent advances in the study of hsp90 structure and mechanism of action. Trends Endocrinol Metab 9: 238-243, 1998.

31. Cardozo CP, Michaud C, Ost MC, et al: C-terminal Hspinteracting protein slows androgen receptor synthesis and reduces its rate of degradation. Arch Biochem Biophys 410: 134-140, 2003.

32. Myzak MC, Karplus PA, Chung FL and Dashwood RH: A novel mechanism of chemoprotection by sulforaphane: inhibition of histone deacetylase. Cancer Res 64: 5767-5774, 2004.

33. Wang LG, Beklemisheva A, Liu XM, Ferrari AC, Feng J and Chiao JW: Dual action on promoter demethylation and chromatin by an isothiocyanate restored GSTP1 silenced in prostate cancer. Mol Carcinog 46: 24-31, 2007.

34. Wang LG, Liu XM, Fang Y, et al: De-repression of the p21 promoter in prostate cancer cells by an isothiocyanate via inhibition of HDACs and c-Myc. Int J Oncol 33: 375-380, 2008. 
35. Wang LG, Liu XM and Chiao JW: Repression of androgen receptor in prostate cancer cells by phenethyl isothiocyanate. Carcinogenesis 27: 2124-2132, 2006.

36. Nakagawa H, Nuovo GJ, Zervos EE, et al: Age-related hypermethylation of the 5' region of MLH1 in normal colonic mucosa is associated with microsatellite-unstable colorectal cancer development. Cancer Res 61: 6991-6995, 2001.

37. Palmisano WA, Divine KK, Saccomanno G, et al: Predicting lung cancer by detecting aberrant promoter methylation in sputum. Cancer Res 60: 5954-5958, 2000.

38. Singal R, van Wert J and Bashambu M: Cytosine methylation repress glutathione S-transferase P1 (GSTP1) gene expression in human prostate cancer cells. Cancer Res 61: 4820-4826, 2001.

39. Costello JF and Plass C: Methylation matters. J Med Genet 38: 285-303, 2001

40. Esteller M: CpG island hypermethylation and tumor suppressor genes: a booming present, a brighter future. Oncogene 21: 5427-5440, 2002.

41. Kopelovich L, Crowell JA and Fay JR: The epigenome as a target for cancer chemoprevention. J Natl Cancer Inst 95: 1747-1757, 2003.

42. Pompeia C, Hodge DR, Plass C, et al: Microarray analysis of epigenetic silencing of gene expression in the KAS-6/1 multiple myeloma cell line. Cancer Res 64: 3465-3473, 2004.

43. Brooks JD, Paton VG and Vidanes G: Potent induction of phase 2 enzymes in human prostate cells by sulforaphane. Cancer Epidemiol Biomarkers Prev 10: 949-954, 2001.

44. Lee WH, Morton RA, Epstein JI, et al: Cytidine methylation of regulatory sequences near the pi-class glutathione S-transferase gene accompanies human prostatic carcinogenesis. Proc Natl Acad Sci USA 91: 11733-11737, 1994.

45. Maruyama R, Toyooka S, Toyooka KO, et al: Aberrant promoter methylation profile of prostate cancers and its relationship to clinicopathological features. Clin Cancer Res 8: 514-519, 2002

46. Woodson K, Gillespie J, Hanson J, et al: Heterogeneous gene methylation patterns among pre-invasive and cancerous lesions of the prostate: a histopathologic study of whole mount prostate specimens. Prostate 60: 25-31, 2004

47. Liu XM, Jiang JD, Ferrari AC, Budman DR and Wang LG: Unique induction of $\mathrm{p} 21$ (WAF1/CIP1) expression by vinorelbine in androgen-independent prostate cancer cells. Br J Cancer 89: 1566-1573, 2003.

48. Fei P, Matwyshyn GA, Rushmore TH and Kong AN: Transcription regulation of rat glutathione S-transferase Ya subunit gene expression by chemopreventive agents. Pharm Res 13: 1043-1048, 1996.

49. Seo KW, Kim JG, Park M, Kim TW and Kim HJ: Effects of phenethyl isothiocyanate on the expression of glutathione Stransferases and hepatotoxicity induced by acetaminophen. Xenobiotica 30: 535-545, 2000.

50. Goessl C, Krause H, Muller M, et al: Fluorescent methylationspecific polymerase chain reaction for DNA-based detection of prostate cancer in bodily fluids. Cancer Res 60: 5941-5945, 2000.
51. Yamada H, Vijayachandra K, Penner $C$ and Glick A: Increased sensitivity of transforming growth factor (TGF) beta 1 null cells to alkylating agents reveals a novel link between TGFbeta signaling and $\mathrm{O}(6)$-methylguanine methyltransferase promoter hypermethylation. J Biol Chem 276: 19052-19058, 2001.

52. Biniszkiewicz D, Gribnau J, Ramsahoye B, et al: Dnmt1 overexpression causes genomic hypermethylation, loss of imprinting, and embryonic lethality. Mol Cell Biol 22: 2124-2135, 2002.

53. McCabe MT, Low JA, Daignault S, Imperiale MJ, Wojno KJ and Day ML: Inhibition of DNA methyltransferase activity prevents tumorigenesis in a mouse model of prostate cancer. Cancer Res 66: 385-392, 2006.

54. Beklemisheva AA, Feng J, Yeh YA, Wang LG and Chiao JW: Modulating testosterone stimulated prostate growth by phenethyl isothiocyanate via $\mathrm{Sp} 1$ and androgen receptor down-regulation. Prostate 67: 863-870, 2007.

55. Craft N, Chhor C, Tran C, et al: Evidence for clonal outgrowth of androgen-independent prostate cancer cells from androgendependent tumors through a two-step process. Cancer Res 59: 5030-5036, 1999.

56. Agus DB, Cordon-Cardo C, Fox W, et al: Prostate cancer cell cycle regulators: response to androgen withdrawal and development of androgen independence. J Natl Cancer Inst 91: 1869-1876, 1999.

57. Rohlff C, Blagosklonny MV, Kyle E, et al: Prostate cancer cell growth inhibition by tamoxifen is associated with inhibition of protein kinase $\mathrm{C}$ and induction of p21(waf1/cip1). Prostate 37: 51-59, 1998.

58. Davies TG, Bentley J, Arris CE, et al: Structure-based design of a potent purine-based cyclin-dependent kinase inhibitor. Nat Struct Biol 9: 745-749, 2002.

59. Bakshi S, Bergman M, Dovrat S and Grossman S: Unique natural antioxidants (NAOs) and derived purified components inhibit cell cycle progression by downregulation of $\mathrm{ppRb}$ and E2F in human PC3 prostate cancer cells. FEBS Lett 573: 31-37, 2004.

60. Wang L, Liu D, Ahmed T, Chung FL, Conaway C and Chiao JW: Targeting cell cycle machinery as a molecular mechanism of sulforaphane in prostate cancer prevention. Int J Oncol 24: $187-192,2004$

61. Chiao JW, Wu H, Ramaswamy G, et al: Ingestion of an isothiocyanate metabolite from cruciferous vegetables inhibits growth of human prostate cancer cell xenografts by apoptosis and cell cycle arrest. Carcinogenesis 25: 1403-1408, 2004.

62. Zhang Y and Talalay P: Anticarcinogenic activities of organic isothiocyanates: chemistry and mechanisms. Cancer Res 54 (Suppl 7): 1976-1981, 1994.

63. Mi L, Siao Z, Hood BL, et al: Covalent binding to tubulin by isothiocyanates: a mechanism of cell growth arrest and apopotosis. J Biol Chem 283: 22136-22146, 2008.

64. Kelly WK, O'Connor OA and Marks PA: Histone deacetylase inhibitors: from target to clinical trials. Expert Opin Investig Drugs 11: 1695-1713, 2002.

65. Jones PA and Baylin SB: The fundamental role of epigenetic events in cancer. Nat Rev Genet 3: 415-428, 2002. 